Mots. Les langages du politique

$91 \mid 2009$

Que devient le pamphlet?

\title{
Henri Boyer éd., Stéréotypage, stéréotypes : fonctionnements ordinaires et mises en scène
}

Aurélie Olivesi

\section{Q OpenEdition}

Journals

Édition électronique

URL : https://journals.openedition.org/mots/19302

DOI : $10.4000 /$ mots. 19302

ISSN : 1960-6001

Éditeur

ENS Éditions

Édition imprimée

Date de publication : 30 novembre 2009

Pagination : 123-128

ISBN : 9782847881820

ISSN : 0243-6450

Référence électronique

Aurélie Olivesi, « Henri Boyer éd., Stéréotypage, stéréotypes : fonctionnements ordinaires et mises en scène », Mots. Les langages du politique [En ligne], 91 | 2009, mis en ligne le 30 novembre 2011 consulté le 22 avril 2022. URL : http://journals.openedition.org/mots/19302 ; DOI : https://doi.org/ $10.4000 /$ mots. 19302

\section{(C) ENS Éditions}




\title{
Comptes rendus de lecture
}

\author{
Stéréotypage, stéréotypes : fonctionnements ordinaires et mises en scène \\ Henri Boyer éd. \\ 2007, Paris, L'Harmattan, 5 tomes, 1230 p.
}

L'ouvrage collectif Stéréotypages, stéréotypes: fonctionnements ordinaires et mises en scène, dirigé par Henri Boyer et rassemblant pas moins de 103 communications, est issu du colloque international qui s'est tenu sur le même thème à l'université Paul Valéry Montpellier 3, du 21 au 23 juin 2006. Ce recueil de cinq tomes ${ }^{1}$ se donne pour but de mettre en lumière les modalités de fonctionnement et les enjeux propres aux stéréotypes, représentations figées toujours disponibles au sein des imaginaires des communautés culturelles. Leur représentation oscille entre deux pôles opposés : si les stéréotypes sont dénoncés par certains en raison de leur nocivité intra- et intercommunautaire, ils sont également considérés comme utiles pour le confort de la communication entre membres de ces communautés.

Les nombreuses contributions de cet ouvrage confrontent des approches provenant de multiples champs disciplinaires - linguistique (sciences du langage, sociolinguistique, sémiotique, analyse du discours, didactologie des langues-cultures), sciences humaines (politologie, sciences de l'information et de la communication, sociocritique, analyse littéraire, étude des discours cinématographiques) et sociales (psychologie sociale, sociologie, ethnologie) - s'incarnant dans une profusion de corpus, d'angles d'attaque et de méthodologies, qui s'attachent à prendre en compte aussi bien les fonctions ordinaires des stéréotypes que leur mise en scène. De même que le colloque était organisé en cinq ateliers, les actes le sont en cinq tomes.

Le premier tome s'intéresse aux stéréotypes et au stéréotypage comme phénomènes communautaires à propos desquels les médias ont, dans les sociétés médiatisées, une responsabilité particulière. Nous étudierons plus précisément les communications publiées dans ce volume qui recoupent particulièrement les champs de recherche de la revue Mots. Les langages du politique. Les corpus analysés dans cette partie appartiennent aux différentes

1. T. I: Média(tisation)s, 298 p.; t. II : Identités, 332 p.; t. III : Éducation, école, didactique, 226 p.; t. IV : Langue(s), discours, 186 p.; t. V : Expressions artistiques, 188 p. 
composantes de l'espace public médiatique : télévision, presse d'information générale, presse spécialisée ou communautaire, publicité. Dix-sept articles sont consacrés à la mise en œuvre des stéréotypes dans le discours de la presse écrite. Cette mise en œuvre présente une tension dans le rôle joué par le discours journalistique dans l'émergence d'une doxa. Vincent Coppola et Odile Camus s'intéressent ainsi à la manière dont la presse écrite véhicule une représentation anxiogène du sida, alors que Ksenija Djordjevic étudie comment la représentation des violences urbaines a repris et exploité les stéréotypes latents dans l'imaginaire de son lectorat. De la même manière, Marceline Chauque met en lumière la manière dont la crise des banlieues de 2005 a donné lieu à une réécriture des stéréotypes véhiculés par les médias. Olivier Pulvar explicite la manière dont les pratiques professionnelles des journalistes établissent un lien entre stéréotypes identitaires et faits d'actualité. Concernant le discours journalistique sur l'immigration, Fathallah Daghmi souligne dans quelle mesure la question relative à l'image de l'immigré dans la presse française a subi un nouvel épisode de stéréotypage à l'occasion du Mondial 1998, où l'immigré devient une chance pour le pays d'accueil. Dans un pays d'immigration comme le Canada, Marie-Ėve Perrot montre le renversement symbolique du rapport de domination, la minorité francophone se trouvant rattachée à l'universalité, via le stéréotype du français comme idéal linguistique universel. La dimension axiologique du stéréotype est également mise en valeur par Vladimir Beliakov, qui révèle dans quelle mesure le stéréotype du pouvoir ne se confond pas avec la notion correspondante, mais est parallèlement doté d'un ensemble de caractéristiques qui relèvent de la catégorie péjorative, représentant le versant négatif de cette notion. L'évolution sémantique, mais également axiologique, d'un stéréotype est analysée par Alexis Blanchet à propos du terme remake : parti du langage cinématographique, ce terme a subi une phase de figement sémantique, puis une autre de défigement en passant dans le domaine des jeux vidéo, pour répondre à l'état et aux enjeux actuels de ce secteur.

Parallèlement, d'autres auteurs s'intéressent plus précisément au dépassement des stéréotypes par le discours journalistique. Éléonore Yasri, en étudiant le discours consacré à la Turquie dans la presse écrite française au cours des mois qui ont précédé l'annonce de l'ouverture des pourparlers d'adhésion avec les responsables de l'Union européenne, s'attache à montrer que la presse quotidienne nationale véhicule (en y adhérant ou non) des représentations ethnostructurelles relativement figées correspondant à l'imaginaire collectif français. Bert Peeters cherche à montrer que, alors que les personnages qui peuplent le fait divers sont souvent stéréotypés, le discours consacré au tueur en série Michel Fourniret s'efforce d'aller au-delà du stéréotype pour en souligner les spécificités. En effet, si le fait-diversier se contentait de rôles stéréotypés, ses récits perdraient bientôt leur attrait. C'est dans cette pers- 
pective que Laurence Leveneur étudie les mythes professionnels stéréotypés associés à la profession journalistique présents dans la presse elle-même.

Plusieurs communications soulignent la présence de cette tension entre exploitation et dépassement des stéréotypes dans le cas de la presse sportive. Jean-Paul Honoré montre l'énonciation paradoxale mise en œuvre dans L'Équipe sur le Japon, dans la mesure où le journal se veut le représentant d'une idéologie humaniste et universaliste, et, parallèlement, dramatise les rivalités entre nations en les essentialisant. On note également une tension entre proximité et posture d'expert dans le commentaire rugbystique, tension entretenue dans l'ethos du commentateur, analysé par Valérie Bonnet. Sandy Montanola, menant une analyse socio-discursive des médias français comme co-construisant une représentation médiatique des boxeuses de haut niveau, montre que le poids des stéréotypes de genre amène à une médiatisation sexuée : soit les sportives sont appréhendées en comparaison avec les normes masculines, soit la construction du discours dénonce les stéréotypes; mais dans ces articles, la boxeuse n'est pas évoquée en tant que sportive et ses performances physiques ne sont pas prises en compte.

Cette tension dans le discours sur les boxeuses se retrouve dans les articles traitant des stéréotypes sur les femmes. Ainsi, Virginie Julliard souligne que la parité n'a pas permis l'émergence de nouveaux modèles politiques positifs. Émilie Moreau s'attache de son côté à montrer dans quelle mesure une «désubversification» des lesbiennes est entreprise par les journalistes, qui se détachent de la représentation de la lesbienne masculine pour représenter les lesbiennes quasi uniquement comme des mères, ce qui ne correspond à aucun stéréotype habituel. Stéphanie Pahud a pour objectif de montrer dans quelle mesure le discours publicitaire, qui jongle en permanence avec les valeurs socioculturelles, fait montre d'une tendance persistante à l'essentialisation des identités sexuées. Ce phénomène est également analysé par Claude Chabrol, qui met en lumière les problèmes de définition posés à propos des concepts de catégorisation et de stéréotype appliqués à la notion de genre (gender). Au croisement de la presse et de la publicité, Caroline Courbières s'attache à montrer dans quelle mesure le discours sur la mode constitue un terrain privilégié pour l'observation des stéréotypes féminins.

La prégnance des stéréotypes dans le discours publicitaire amène plusieurs auteurs à s'intéresser spécifiquement à ce discours. Stéphanie Perrouty-Perret livre ainsi une analyse discursive sur les descriptions textuelles des odeurs de produits d'entretien, dont les parfums ne sont généralement pas explicités, mais, au contraire, exprimés de manière métaphorique afin de guider l'imaginaire des consommateurs vers des représentations suggestives. Or, les stéréotypes liés aux fragrances sont remarquablement présents au sein des propos des consommateurs, ce qui suggère un mimétisme manifeste entre les productions langagières de ces derniers et les discours publicitaires élaborés par les 
marques. L'approche métaphorique est également privilégiée dans une perspective sociolinguistique par Maria-Rosaria Dagostino et Estelle Lebel, qui cherchent à définir le stéréotype visuel dans la publicité. Montserrat López Díaz montre comment la publicité joue à la fois sur le recours aux idées reçues et la subversion des stéréotypes pour exercer un impact sur l'esprit du consommateur. Dylia Lysardo-Dias, de son côté, cherche à identifier et analyser les modes de fonctionnement discursif et les univers de référence des stéréotypes visuels dans la publicité brésilienne. L'aspect fonctionnel du stéréotypage est montré par Constance et Aurore Adinsi à propos de la campagne télévisuelle de lutte contre la discrimination de 2002. Le recours à des situations stéréotypées peut s'expliquer par le consensus nécessaire à l'articulation de plusieurs milieux de travail.

Enfin, un article s'attache à une approche métadiscursive des stéréotypes : Estelle Lebel, Marguerite Lavallée, Marie-Ève Girard et Amélie Descheneau montrent que la réception télévisuelle par les enfants se révèle en réalité à double ressort, car la communication médiatique se trouve ensuite médiatisée par le groupe de pairs et c'est dans cette perspective que le stéréotype fonctionne comme un ciment social.

Cette approche fonctionnelle des stéréotypes se retrouve dans le deuxième tome, consacré au rôle des stéréotypes dans la catégorisation (groupale, sexuelle, sociale...) et la construction ou la (re)production des identités. Ce volume s'attache conjointement aux identités revendiquées et aux identités imposées. Ainsi, sept articles sont consacrés à la représentation d'un « autre » ethnique ou culturel à des fins promotionnelles (le Canada dans une foire internationale pour Gabriele Budach, Alexandre Duchêne, Claudine Moïse et Mary Richards, le Maroc à travers ses brochures touristiques pour Patricia Caille et Pierre-Alain Mallet, et les «Français romantiques» dans l'imaginaire chinois pour Sylvain Dreyer), argumentatives (la «provocation turque» servant à la construction discursive d'une vision nationale roumaine et française pour Valentina Pricopie, ou la formulation de «Sud» qui, sous des apparences simplement toponymiques, constitue un stéréotype discursif implicite dans le cadre de la reformulation d'une stéréotypie relationnelle ancrée dans une histoire de la dominance coloniale, comme le montre Françoise Dufour), ou de stigmatisation (Abdenbi Lachkar étudie ainsi les représentations et relations intergroupales dans les villes marocaines à travers des locutions expressives particulièrement présentes dans la phraséologie fassie, et de la même manière, Jean-Léo Léonard s'attache à montrer comment les stéréotypes hérités du passé colonial sont utilisés dans les conflits entre castes, classes et groupes humains au Guatemala), au point de servir de révélateur identitaire dans le cas d'un pays à l'identité nationale fragmentée comme la Suisse (Sara Cotelli mais également Stefano Losa). C'est dans sa dimension interactionnelle que le recours aux stéréotypes prend son sens. Ainsi l' "utilité » des sté- 
réotypes est-elle explicitée dans le domaine politique (Aurélie Morin montre à partir d'entretiens les stratégies élaborées par les candidats d'origine maghrébine pour se détacher du stéréotype de «l'arabe de service»), dans celui de l'homosexualité (où Caroline Dayer se demande à quel moment un stéréotype devient nocif), ou pour justifier un ordre inégalitaire établi, comme c'est le cas pour les stéréotypes de genre (sexotypes pour Sophie Bailly, rapports professionnels hommes/femmes pour Pascale Molinier). On note également un phénomène de réhabilitation par le biais des stéréotypes dans le cas du dauphin, dont l'image devient plus positive à mesure qu'émerge une conscience écologique, comme le souligne Emmanuel Gouabault.

La dimension interactionnelle est présente dans le troisième tome, consacré à l'approche didactique des stéréotypes dans le domaine éducatif - particulièrement dans l'enseignement et l'apprentissage des languescultures -, et qui s'attache principalement à établir une distinction entre le stéréotype comme représentation collective figée et le stéréotypage comme processus cognitif. Ce volume se donne pour objectif de montrer dans quelle mesure cette distinction peut être féconde pour toute réflexion sur les stéréotypes culturels, qu'il s'agisse des phénomènes d'apprentissage de la lecture (Jean-Louis Dufays), l'étude des «genres» cinématographiques dans l'enseignement secondaire français actuel (Philippe Bourdier), l'apprentissage du français comme langue étrangère (Nathalie Auger, Phyllis Dalley et Sylvie Roy; Margaret Bento ; Fred Dervin et Eija Suomela-Salmi) ou des questions liées au plurilinguisme (Karima Ait Dahmane; Stéphanie Clerc).

Le quatrième tome est consacré aux stéréotypes dans la langue et le discours - discours épilinguistiques, actes de communication. II s'agit, pour les auteurs, soit d'analyser en quoi le stéréotype dans la langue sert d'outil d'observation de la société (Soufian Al Karjouski étudie le rôle des stéréotypes dans l'effacement de la polysémie dans la langue arabe, Julien Longhi et Georges Elia Sarfati la dénomination intermittent et Ida Machado la représentation de la prostituée), soit, par une approche sémantico-discursive, de comprendre le rôle des stéréotypes dans la structure de la signification lexicale, qu'il s'agisse de leur rôle dans les conflits diglossiques, comme l'analyse Henri Boyer, ou de l'étude menée par Olga Galatanu sur le décalage entre l'aspect évaluatif du langage ordinaire et les aspects dénotatifs de son usage en sciences sociales.

Le cinquième et dernier tome traite du rapport ambigu des stéréotypes à l'expression artistique, notamment la littérature et le cinéma. Comment, en effet, l'artiste se réapproprie-t-il le schème collectif du stéréotype pour construire une œuvre originale? L'usage des stéréotypes dans les œuvres artistiques a pour fonction principale de jouer sur la distance et la proximité (Évelyne Argaud; Catherine Berthet-Cahuzac; Géronimo Potocnjak). Mais leur usage peut être plus conscient, qu'il s'agisse du recours à un sujet 
dépersonnalisé, comme chez Nathalie Sarraute (Renato De Mello), ou à un archétype, comme pour Salvador Dali (Jean-Antoine Diaz). À l'encontre des représentations traditionnelles, Alphonse J. Tonyè montre l'importance du stéréotype comme élément de pérennisation des coutumes multiséculaires dans le roman francophone négro-africain. Enfin, dans le discours portant sur l'art, l'usage des stéréotypes présente un effet didactique de compréhension immédiate (Françoise Rigat pour les catalogues d'exposition, Jean Szlamowicz pour le jazz, Bernard Tabuce pour «La Guerre des Demoiselles»).

Face à la richesse de la matière exposée dans cet ouvrage collectif, on regrettera simplement la rareté des balises permettant la circulation au sein même de cette matière. La proximité de certains thèmes traités et de certains éclairages méthodologiques dans des articles relativement éloignés les uns des autres dans l'économie générale de l'ouvrage aurait pu être soulignée par une préface propre à chaque volume qui en précisât le contenu, les spécificités et les enjeux, et par une table des matières hiérarchisée qui permît d'en appréhender rapidement les principales thématiques, ou un index. Or, les liens entre les différents articles appartenant aux divers tomes de cet ouvrage ne se découvrent qu'au hasard d'une lecture linéaire, paradoxale dans un ouvrage collectif, qui appellerait plutôt une lecture transversale.

Aurélie Olivesi

Université Toulouse 2 Le Mirail

aurelie.olivesi@wanadoo.fr 